\title{
Triple Oral Antithrombotic Therapy in Atrial Fibrillation and Coronary Artery Stenting: Searching for the Best Combination
}

\author{
Hazem Elewa, RPh, PhD, BCPS ${ }^{1}$ Dina Ahmed, PharmD² Geoffrey D. Barnes, MD, MSc ${ }^{3}$ \\ ${ }^{1}$ Department of Clinical Pharmacy and Practice, College of Pharmacy, \\ Qatar University, Doha, Qatar \\ 2 Hamad General Hospital, Hamad Medical Corporation, Doha, Qatar \\ ${ }^{3}$ Frankel Cardiovascular Center and Institute for Healthcare Policy and

\begin{abstract}
Address for correspondence Geoffrey D. Barnes, MD, MSc, 2800 Plymouth Rd. Building 14 Room G101, Ann Arbor, MI 48109-2800 (e-mail: gbarnes@umich.edu).
\end{abstract} Innovation, University of Michigan, Ann Arbor, Michigan

Semin Thromb Hemost 2016;42:662-670.

\begin{abstract}
Patients with atrial fibrillation (AF) who are treated with oral anticoagulants often have concurrent coronary artery disease. Triple oral antithrombotic therapy (TOAT) is often necessity to prevent stent thrombosis or myocardial infarction associated with percutaneous coronary intervention or acute coronary syndrome in patients with comorbid coronary artery disease and AF. Although the use of TOAT (aspirin, clopidogrel, and warfarin) has excellent efficacy against thrombotic complications, this comes on the expense of increased bleeding risk. This review discusses potential strategies to improve TOAT benefit-risk ratio evidence from the literature. These strategies include: (1) dropping aspirin; (2) reducing the duration of TOAT; (3) switching warfarin to a direct

Keywords

- antithrombotic

- antiplatelet

- warfarin

- atrial fibrillation

- acute coronary syndrome oral anticoagulant (DOAC); (4) the use of DOAC in combination with a single antiplatelet agent; and (5) switching clopidogrel to a novel antiplatelet agent. Although dropping aspirin and reducing TOAT duration should be considered in selected AF patients at low risk of thrombosis, the role of DOACs and novel antiplatelets in TOAT has not been thoroughly studied, and there is limited evidence to support their use currently. Ongoing studies will provide safety and efficacy data to guide clinicians who frequently face the challenge of determining the best TOAT combination for their patients.
\end{abstract}

\section{Stroke Prevention in Atrial Fibrillation Using Oral Anticoagulants}

Atrial fibrillation (AF) is one of the most common cardiac arrhythmias, with $10 \%$ prevalence in patients $\geq 80$ years of age. ${ }^{1-3} \mathrm{AF}$ is an independent risk factor for stroke with an annual risk ranging from 2 to $18 \%$ based on the presence of other risk factors. Among different oral antithrombotic therapy studied, warfarin was shown to reduce stroke risk by $64 \%$ when compared with placebo and by $39 \%$ when compared with aspirin in AF patients. ${ }^{4}$ Furthermore, it has been shown to be more effective than dual antiplatelets (DAP) when tested as an alternative antithrombotic regimen. ${ }^{5}$ Although the effectiveness of warfarin was evident, the safety and complexity of managing warfarin therapy left patients and practitioners searching for alternative treatment options. Since 2010, four direct oral anticoagulants (DOACs) have been introduced into the market as an alternative to warfarin. DOACs act by directly and selectively inhibiting key coagulation factors such as thrombin (i.e., dabigatran) or factor Xa (i.e., rivaroxaban, apixaban, and edoxaban). ${ }^{6-8}$ For many nonvalvular AF patients, DOACs are now recommended as first-line therapy for stroke prevention due to a favorable side effect profile and a lower risk of intracerebral hemorrhage. ${ }^{9,10}$ In addition, these agents do not usually require strict routine monitoring or dose adjustments and have fewer drug-drug and drug-food interactions. published online May 28, 2016
Issue Theme Editorial Compilation II; Guest Editors: Emmanuel J. Favaloro, PhD, FFSc (RCPA), and Giuseppe Lippi, MD.
Copyright @ 2016 by Thieme Medical Publishers, Inc., 333 Seventh Avenue, New York, NY 10001, USA. Tel: +1(212) 584-4662.
DOI http://dx.doi.org/ 10.1055/s-0036-1571337. ISSN 0094-6176. 


\section{Ischemic Risk Prevention in Patients with Acute Coronary Syndromes and/or Undergoing Percutaneous Coronary Intervention Using DAP Treatment}

Since 2001, DAP treatment with aspirin and clopidogrel has been the treatment of choice in patients with acute coronary syndromes (ACS) and in patients undergoing percutaneous coronary intervention ( $\mathrm{PCI}$ ) to prevent complications such as stent thrombosis, recurrent myocardial infarction (MI), and stroke. ${ }^{11,12}$ Although aspirin is known to significantly reduce cardiovascular events after ACS, the addition of clopidogrel as a second antiplatelet was found to improve ACS outcomes significantly compared with aspirin alone. ${ }^{13,14}$ Not long ago, novel antiplatelet agents (prasugrel and ticagrelor) were introduced as alternatives to clopidogrel in the setting of ACS. Prasugrel and ticagrelor generally achieve higher degrees of platelet inhibition than clopidogrel and do not appear to be affected by CYP2C19 polymorphism. ${ }^{15,16}$ As compared with clopidogrel, both agents were shown to improve clinical outcomes among patients with ACS but with an increase in bleeding risk, particularly in those undergoing PCI. ${ }^{17,18}$ Current guidelines recommend the use of DAP for at least 4 weeks after bare-metal stent and for at least 6 months after drug-eluting stent (DES). ${ }^{19,20}$ However, the exact duration of DAP therapy in patients receiving DES placement remains a controversial issue. ${ }^{21-23}$

\section{Triple Oral Antithrombotic Therapy}

Triple oral antithrombotic therapy (TOAT) is commonly used for patients with AF on oral anticoagulant therapy who experience an ACS or undergo elective PCI. It is estimated that coronary artery disease coexists in 20 to $30 \%$ of AF patients who also qualify for oral anticoagulant therapy. ${ }^{24,25}$ Aspirin, clopidogrel, and warfarin have long been the mainstay TOAT combination. Despite its adequate efficacy, suboptimal safety remains a topic of much concern, especially given the morbidity and mortality associated with antithromboticrelated bleeding. ${ }^{26-30}$ Potential strategies to improve TOAT (aspirin, clopidogrel, and warfarin) benefit-risk ratio include: (1) dropping aspirin; (2) reducing the duration of TOAT; (3) switching warfarin to a DOAC; (4) the use of DOAC in combination with a single antiplatelet agent; and (5) switching clopidogrel to a novel antiplatelet agent ( - Table 1 ). In this review, we will discuss evidence from the literature on the efficacy, safety, and usefulness of these different strategies (-Table 2).

\section{Dropping Aspirin}

The reported efficacy of the TOAT (aspirin, clopidogrel and warfarin) comes at the expense of increased risk of bleeding. Several observational studies reported two- to threefold increase in bleeding with the use of TOAT when compared with DAP or to the use of warfarin and a single antiplatelet agent. $^{27,29,31,32}$ Since warfarin was deemed necessary for stroke prevention and the omission of clopidogrel could
Table 1 Potential strategies to improve TOAT (aspirin, clopidogrel, and warfarin) benefit-risk ratio

\begin{tabular}{|l|}
\hline 1. Dropping aspirin \\
\hline 2. Reducing the duration of TOAT \\
\hline 3. Switching warfarin to a DOAC \\
\hline $\begin{array}{l}\text { 4. The use of DOAC in combination with a single } \\
\text { antiplatelet agent }\end{array}$ \\
\hline 5. Switching clopidogrel to a novel antiplatelet agent \\
\hline
\end{tabular}

Abbreviations: DOAC, direct oral anticoagulant therapy; TOAT, triple oral anticoagulant therapy.

lead to an increased risk of stent thrombosis, the combination of warfarin and clopidogrel was chosen by The WOEST (The What is the Optimal antiplatElet and anticoagulant therapy in patients with oral anticoagulation and coronary StenTing) study investigators to be tested for its safety and efficacy against TOAT $^{30}$ This randomized controlled trial of 573 patients with ACS or elective PCI and a concurrent need for long-term warfarin therapy demonstrated that patients randomized to warfarin plus clopidogrel (omitting aspirin) had significantly reduced risk of major bleeding compared with patients randomized to TOAT therapy (19.4 vs. $44.4 \%$; hazard ratio [HR], 0.36; 95\% confidence interval $[\mathrm{Cl}], 0.26-0.40$; $p<0.001)$. In addition, the use of clopidogrel with warfarin alone (omitting aspirin) was associated with a lower risk of combine thromboembolic endpoint that included death, MI, stroke, target-vessel revascularization, and stent thrombosis (11.1 vs. $17.6 \%$; HR, $0.60 ; 95 \% \mathrm{Cl}, 0.38-0.94 ; p=0.025$ ). However, as the trial was powered to detect a difference in major bleeding and not in thromboembolic events, there were no statistically significant differences seen between the two study groups for MI, target-vessel revascularization, stroke, or stent thrombosis. All-cause mortality rate was lower in the clopidogrel/warfarin group compared with the TOAT group, which could be mediated by the lower number of bleeding in the clopidogrel/warfarin group. Similar findings were also reported in recently published large datasets highlighting the increased risk of bleeding and lack of clinical benefit associated with TOAT compared with warfarin/single antiplatelet. ${ }^{33-35}$

\section{Reducing the Duration of Triple Oral Antithrombotic Therapy}

In patients receiving a stent post-ACS/PCI without an indication for oral anticoagulant, shortening the duration of DAP therapy has been studied extensively. These trials yielded mixed results regarding the net clinical efficacy but reached agreement on the increased risk of bleeding with prolonged DAP therapy. ${ }^{23,36,37}$ Similarly, longer duration of TOAT has long been recognized to increase the risk of bleeding complications. Recently, the ISAR-TRIPLE (Duration of Triple Therapy in Patients Requiring Oral Anticoagulation After Drug-Eluting Stent Implantation) study investigated TOAT after stenting and its duration. ${ }^{38}$ Its aim was to evaluate 


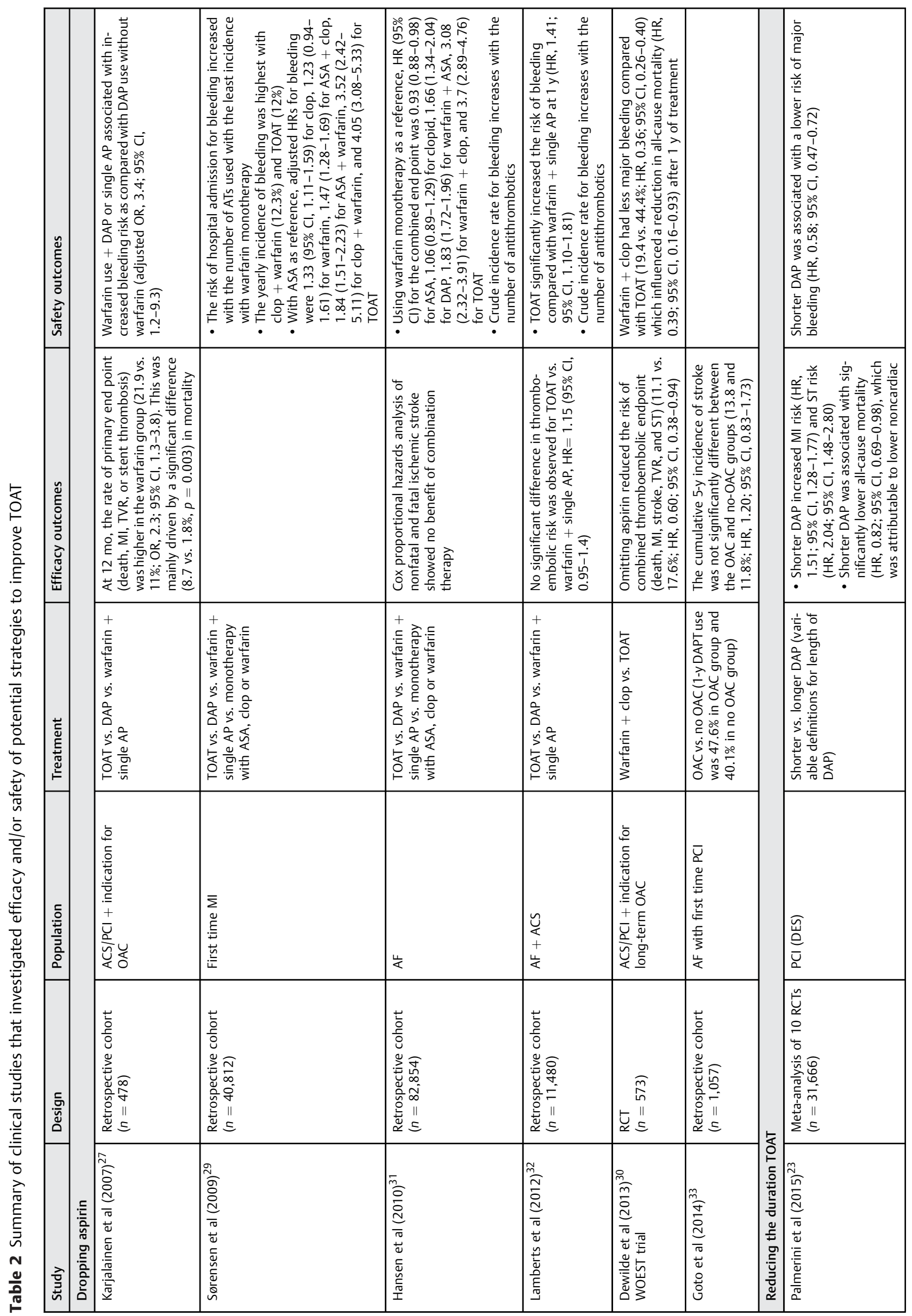




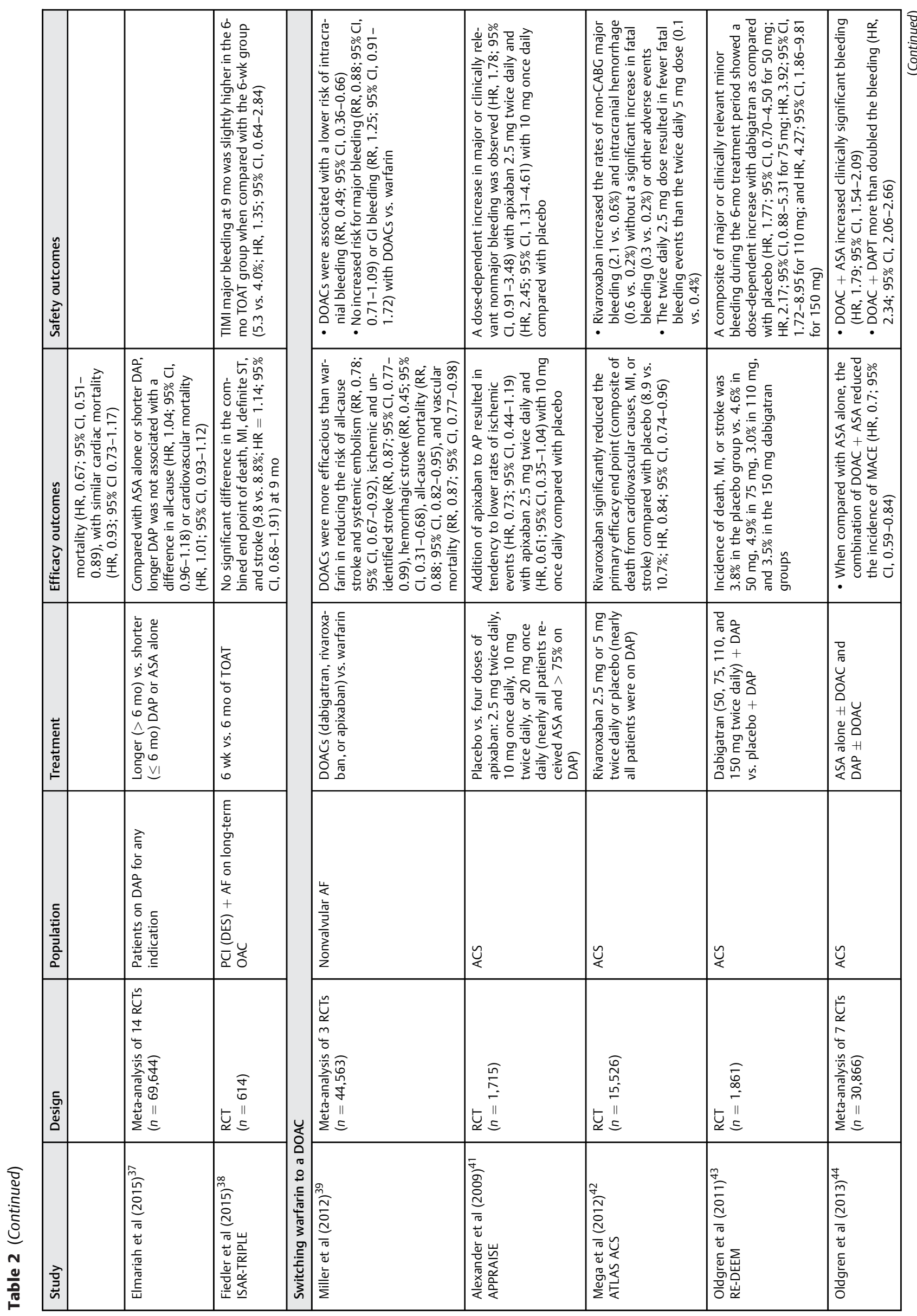




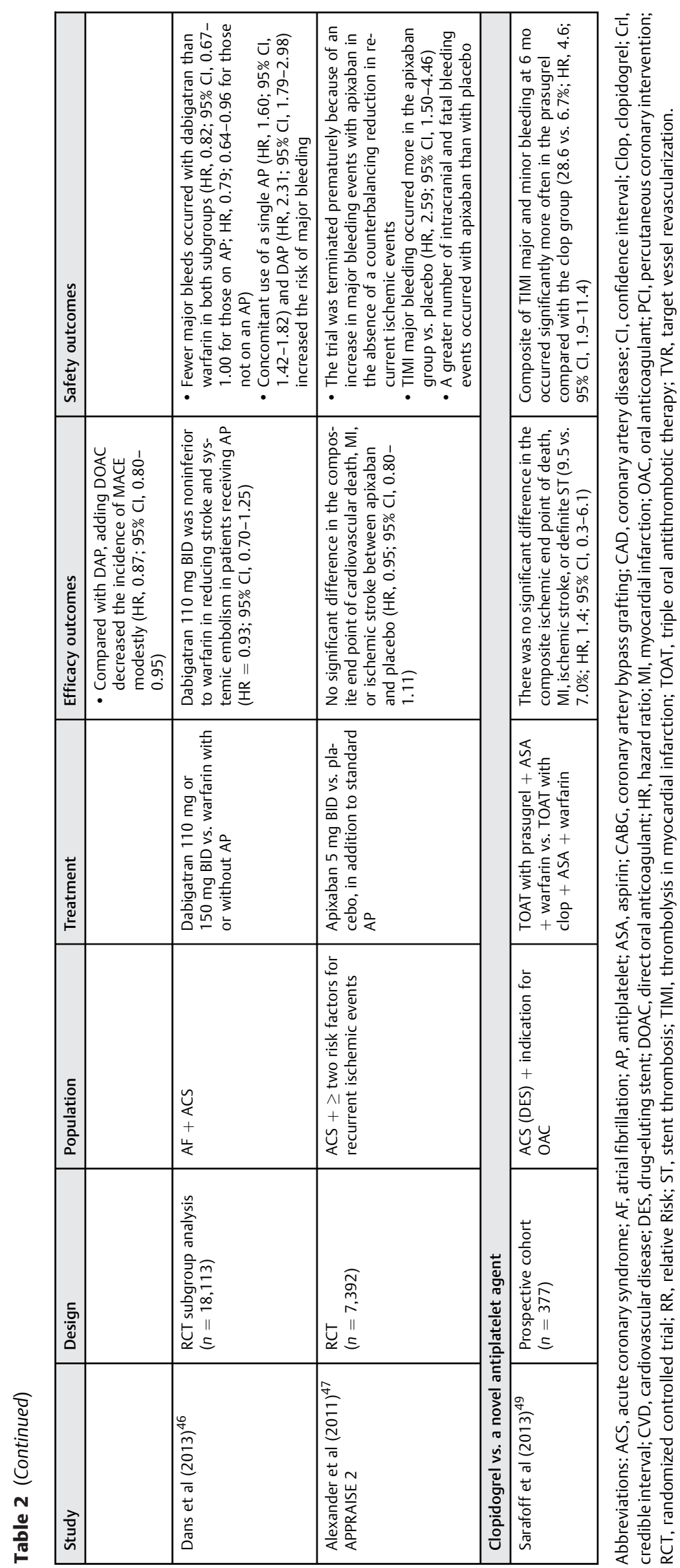


whether shortening the duration of clopidogrel therapy from 6 months to 6 weeks after DES implantation was associated with a superior net clinical outcome in patients receiving concomitant aspirin and oral anticoagulant therapy. This study demonstrated that among 614 patients on long-term oral anticoagulation who are receiving DES for stable angina or ACS, a 6-week TOAT course was not superior to a 6-month triple therapy. Specifically, there was no significant difference in the primary combined end point of death, MI, definite stent thrombosis, and stroke (9.8 vs. $8.8 \%$; $\mathrm{HR}=1.14 ; 95 \% \mathrm{CI}$, $0.68-1.91 ; p=0.63$ ), or the secondary bleeding end point of thrombolysis in MI (TIMI) major bleeding (5.3 vs. $4.0 \%$; HR, $1.35 ; 95 \% \mathrm{CI}, 0.64-2.84 ; p=0.44)$ in the 6 -month group when compared with the 6-week group at 9 months. Individual components of the primary end point were similar, with the exception of a higher incidence of MI in the 6-week compared with the 6 -month therapy group ( 2 vs. $0 \% ; p=0.03$ ). However, results of this trial should be interpreted in light of several limitations. First, the difference in the incidence of MI is likely due to imbalance in the ischemic events between groups at 6 weeks when both arms were still on TOAT. Only one MI event occurred after 6 weeks (on day 212 post-PCI) while the patient was receiving oral anticoagulation and aspirin. This imbalance is likely due to the early randomization of patients' post-PCl and not at 6 weeks when prior event exposure might have been balanced by randomization. ${ }^{38}$ Second, the study was only powered to detect a fairly large reduction (60\%) in events, leaving the subgroups underpowered for further analyses. Apart from the ISAR-TRIPLE trial, shortening the duration of clopidogrel in TOAT-treated patients has not been studied prospectively. A retrospective nationwide registry found no clinical benefit and possible harm with the prolonged use of TOAT beyond 4 months. ${ }^{33}$

\section{Switching Warfarin to a Direct Oral Anticoagulant}

DOACs have repeatedly reduced the risk of intracerebral bleeding when compared with warfarin in several phase III studies. ${ }^{39}$ Therefore, replacement of warfarin with a DOAC in TOAT is a logical strategy to reduce the risk of bleeding in patients requiring combined anticoagulant and antiplatelet therapy. Unlike warfarin, DOACs have fast onset of action and do not have fluctuating levels of anticoagulant effect except in patients with sudden deterioration of kidney and/or renal function, as well as in those receiving antibiotic therapy. ${ }^{40}$ Despite these benefits, the exact role of DOACs in patients with ACS and AF is not fully elucidated. AF patients requiring oral anticoagulant therapy were systematically excluded from recent ACS trials testing DOACs, and patients with recent ACS were excluded from most phase III stroke prevention trials in AF patients. ${ }^{41-45}$ So far, there have been no published head-to-head comparisons of any DOACs and warfarin in AF patients with ACS except for a subanalysis of the RE-LY study ${ }^{46}$ This substudy showed that dabigatran $110 \mathrm{mg}$ BID was noninferior to warfarin in reducing stroke and systemic embolism and was associated with fewer major bleeds regardless of the use of DAP. However, the effect of dabigatran
$150 \mathrm{mg}$ BID on stroke and systemic embolism reduction was less prominent among patients using DAP. In addition, dabigatran $150 \mathrm{mg}$ had similar rate of major bleeding to warfarin regardless of the use of DAP. In the time-dependent analysis, there was an additive effect on major bleeding risk with the number of antiplatelets used regardless of the oral anticoagulant dose used ( $\mathrm{HR}, 1.60 ; 95 \% \mathrm{CI}, 1.42-1.82$ for a single vs. no antiplatelet and HR, 2.31; $95 \% \mathrm{CI}, 1.79-2.98$ for DAP vs. no antiplatelet). It is important to remember that this post hoc analysis was not part of the primary randomization and therefore must be interpreted as an observational (nonrandomized) finding.

The use of DOACs in combination with aspirin and clopidogrel was more commonly studied in patients with ACS (but not AF). For instance, in the Re-DEEM study, the addition of different doses of dabigatran or placebo to DAP treatment post-ACS was assessed in 1,861 patients. ${ }^{43}$ This phase II study showed that dabigatran was associated with a dose-dependent increase in bleeding events when compared with placebo for $50 \mathrm{mg}$ (HR, 1.77; 95\% CI, 0.70-4.50); for $75 \mathrm{mg}$ (HR, 2.17; 95\% Cl, 0.88-5.31); for $110 \mathrm{mg}(\mathrm{HR}, 3.92$; $95 \% \mathrm{Cl}$, $1.72-8.95)$; and for $150 \mathrm{mg}$ ( $\mathrm{HR}, 4.27$; $95 \% \mathrm{CI}, 1.86-9.81$ ), with no significant difference in the cardiovascular efficacy outcomes. On the contrary, low-dose rivaroxaban (2.5 or $5 \mathrm{mg}$ BID) were compared with placebo in 15,526 ACS patients receiving DAP treatment in ATLAS ACS 2-TIMI 51 study. ${ }^{42}$ Both rivaroxaban doses were shown to significantly improve the primary cardiovascular efficacy outcomes (9.1 vs. $10.7 \%$; HR, 0.84 ; $95 \% \mathrm{CI}, 0.72-0.97$ for $2.5 \mathrm{mg}$ and 8.8 vs. $10.7 \%$; HR, 0.85 ; $95 \% \mathrm{CI}, 0.73-0.98$ for $5 \mathrm{mg}$ ). A reduction in cardiovascular and all-cause mortality was also shown in the rivaroxaban $2.5 \mathrm{mg}$ arm (2.7 vs. $4.1 \%$; HR, 0.66 ; $95 \% \mathrm{Cl}, 0.51-0.86$ for cardiovascular mortality and 2.9 vs. $4.5 \%$; $\mathrm{HR}, 0.83 ; 95 \% \mathrm{CI}$, 0.72-0.97 for all-cause mortality). However, major bleeding and intracranial hemorrhage were significantly higher in the rivaroxaban group (2.1 vs. $0.6 \%$; $\mathrm{HR}, 3.96 ; 95 \% \mathrm{CI}, 2.46-6.38$ for major TIMI bleeding and 0.6 vs. $0.2 \%$; $\mathrm{HR}, 3.28 ; 95 \% \mathrm{CI}$, 1.28-8.42 for intracranial hemorrhage), without a significant increase in fatal bleeding. Finally, a range of apixaban doses compared with placebo in ACS patients receiving DAP treatment were investigated in the APPRAISE trial. ${ }^{41}$ In this study, apixaban $10 \mathrm{mg}$ BID and apixaban $20 \mathrm{mg}$ once daily were discontinued because of excess total bleeding. The two other doses of apixaban (2.5mg BID and $10 \mathrm{mg}$ once daily) still showed a dose-related increase in bleeding and a trend toward improvement in cardiovascular ischemic events. Apixaban $5 \mathrm{mg}$ BID was then explored further in the APPRAISE-2 study. ${ }^{47}$ The study was prematurely stopped due to excessive TIMI major bleeding in the apixaban group (1.3 vs. $0.5 \%$; HR, 2.59 ; $95 \% \mathrm{CI}, 1.50-4.46 ; p=0.001$ ) without a significant improvement in the efficacy outcomes.

A recent meta-analysis, including seven randomized, placebo-controlled phase II and III studies of DOACs in 30,866 patients with recent ACS, showed that the addition of DOACs to aspirin alone led to a 30\% reduction in major adverse cardiovascular events (MACE) (HR, 0.70; 95\% Cl, 0.59-0.84), but a substantial increase in bleeding (HR, 1.79; 95\% CI, 1.54-2.09). The reduction in MACE events was attenuated 
(HR, 0.87; 95\% CI, 0.80-0.95) and the risk of major bleeding was more pronounced (HR, 2.34; 95\% CI, 2.06-2.66) when DOACs were used in combination with DAP therapy including aspirin and clopidogrel. ${ }^{44}$

\section{Use of Direct Oral Anticoagulant in Combination with a Single Antiplatelet Agent}

To explore the outcomes associated with DOAC therapy as a part of the antithrombotic therapy in AF patients who experience an ACS, two trials are ongoing or planned. The first trial, PIONEER AF-PCI (NCT01830543), is an open-label, randomized, controlled, multicenter study exploring rivaroxaban or dose-adjusted warfarin in subjects with AF who undergo PCI and require DAP therapy. ${ }^{48}$ This trial is designed to explore safety outcomes, primarily related to clinically significant bleeding. This trial will also study the more potent platelet inhibitors prasugrel and ticagrelor in combination with oral anticoagulant with regard to bleeding safety outcomes. A similar but larger clinical trial with dabigatran is the RE-DUAL PCI (Evaluation of Dual Therapy With Dabigatran versus Triple Therapy With Warfarin in Patients With AF That Undergo a PCI With Stenting) trial (NCT02164864). The main objective of this study is to compare a dual antithrombotic therapy regimen of dabigatran $110 \mathrm{mg}$ BID plus clopidogrel to a TOAT combination of warfarin plus clopidogrel or ticagrelor plus aspirin in AF patients who undergo a PCI with stenting (elective or due to ACS). The study aims to demonstrate noninferiority of dabigatran when compared with warfarin in efficacy and safety. However, it is important to note that both trials will not evaluate the combination of DOACs with DAP but will evaluate the efficacy and safety of dual therapy of DOAC combined with a single antiplatelet to TOAT with warfarin, aspirin, and clopidogrel. ${ }^{11}$

\section{Switching Clopidogrel to a Novel Antiplatelet Agent}

Although the previously discussed strategies were intended primarily to reduce the risk of bleeding associated with TOAT, switching clopidogrel to a novel antiplatelet agent aims to enhance the net clinical benefit rather than reducing the risk of bleeding. Novel antiplatelet drugs, such as prasugrel and ticagrelor, have been shown to be more effective at reducing recurrent MI, stroke, and death than clopidogrel in patients with ACS, but they were also associated with an increased risk of bleeding. ${ }^{17,18}$ The use of prasugrel in TOAT was studied by Sarafoff et al in 2013. ${ }^{49}$ When compared with clopidogrel, the treatment with prasugrel in addition to aspirin and warfarin for a 6-month regimen was associated with a significant increase in the rate of bleeding (28.6 vs. $6.7 \%$; HR, 3.2; 95\% CI, 1.1-9.1; $p=0.03$ ). There was no significant difference in the combined ischemic secondary end points. Similarly, there was a recent report of two cases of fatal bleeding when prasugrel was used as part of TOAT. ${ }^{50}$ To that end, use of prasugrel is generally not recommended as a part of a TOAT regimen. ${ }^{19}$ Although ticagrelor was not studied to date in TOAT, it is likely to expose patients to a higher risk of bleeding as well given its results in ACS patients when compared with clopidogrel. ${ }^{17}$

\section{Guideline Recommendations}

Both the American College of Cardiology/American Heart Association (AHA) and the European Society of Cardiology (ESC) recommend shortening the duration of TOAT as much as possible when indications dictate the use of all three antithrombotic agents. ${ }^{19,51}$ They then recommend the use of an oral anticogulant plus a single antiplatelet agent to reduce bleeding risk. The TOAT regimen recommended by the ESC is aspirin and clopidogrel combined with either warfarin or the lowest dose of DOAC (dabigatran $110 \mathrm{mg}$ BID, rivaroxaban $15 \mathrm{mg}$ daily, and apixaban $2.5 \mathrm{mg}$ BID). The AHA, however, does not recommend the use of DOACs in TOAT since they were not evaluated in AF patients with ACS/PCI. The bleeding risk associated with prasugrel is excessive and was not tested for ticagrelor, hence both the AHA and the ESC recommend against the use of novel antiplatelets in TOAT. Both guidelines also recommend to maintain international normalized ratio levels between 2.0 and 2.5 in AF patients receiving warfarin along with DAP. ${ }^{52}$ A summary of both guidelines' recommendations is provided in - Table 3 .

Table 3 ACC/AHA and ESC guideline summary on the use of TOAT

\begin{tabular}{|l|l|}
\hline Guideline & Recommendation \\
\hline ACC/AHA and ESC & $\begin{array}{l}\text { Shorten the duration of TOAT as much as possible followed by the use of oral anticoagulant and a single } \\
\text { antiplatelet agent }\end{array}$ \\
\hline ACC/AHA & Recommended TOAT: warfarin + aspirin + clopidogrel \\
\hline ESC & $\begin{array}{l}\text { Recommended TOAT: warfarin or the lowest dose of DOAC (dabigatran } 110 \mathrm{mg} \text { BID, rivaroxaban } \\
15 \mathrm{mg} \text { daily, apixaban } 2.5 \mathrm{mg} \text { BID) }+ \text { aspirin + clopidogrel }\end{array}$ \\
\hline ACC/AHA and ESC & Control INR levels between 2.0 and 2.5 in AF patients receiving warfarin along with DAP \\
\hline ACC/AHA and ESC & Recommend against the use of novel antiplatelets in TOAT \\
\hline
\end{tabular}

Abbreviations: ACC/AHA, American College of Cardiology/American Heart Association; AF, atrial fibrillation; DAP, dual antiplatelets; DOAC, direct oral anticoagulant; ESC, European Society of Cardiology; INR, international normalized ratio; TOAT, triple oral antithrombotic therapy. 


\section{Summary}

TOAT (warfarin/aspirin/clopidogrel) increases the risk of bleeding when compared with the use of a combination of warfarin and a single antiplatelet agent. Dual therapy with warfarin and clopidogrel should be considered as an alternative to triple therapy in selected AF patients at low risk of stent thrombosis/recurrent cardiac events. If TOAT (warfarin/aspirin/clopidogrel) therapy is used, clinicians must weigh ischemic and bleeding risks of their patients when deciding on the duration of this combination due to the fact that its optimal duration is not established. The use of prasugrel in combination with warfarin and aspirin leads to a significant increase in bleeding compared with the combination of clopidogrel, aspirin, and warfarin. Therefore, the use of prasugrel (or ticagrelor) as a part of TOAT is not recommended. ${ }^{19}$

Among patients with ACS but no comorbid AF, the addition of a DOAC to antiplatelet therapy led to a modest reduction in cardiovascular events but on the expense of excessive bleeding risk. This was most evident in patients receiving DAP therapy. Notably, the reduction in ischemic events by DOACs was most promising when added to a single antiplatelet therapy. Therefore, studies evaluating the combination of DOACs and a single antiplatelet therapy in AF patients who experience an ACS were initiated and results are still pending.

\section{References}

1 Go AS, Hylek EM, Phillips KA, et al. Prevalence of diagnosed atrial fibrillation in adults: national implications for rhythm management and stroke prevention: the AnTicoagulation and Risk Factors in Atrial Fibrillation (ATRIA) Study. JAMA 2001;285(18): 2370-2375

2 Kannel WB, Benjamin EJ. Status of the epidemiology of atrial fibrillation. Med Clin North Am 2008;92(1):17-40, ix i x.

3 Stewart S, Hart CL, Hole DJ, McMurray JJ. Population prevalence, incidence, and predictors of atrial fibrillation in the Renfrew/ Paisley study. Heart 2001;86(5):516-521

4 Hart RG, Pearce LA, Aguilar MI. Meta-analysis: antithrombotic therapy to prevent stroke in patients who have nonvalvular atrial fibrillation. Ann Intern Med 2007;146(12):857-867

5 Connolly S, Pogue J, Hart R, et al; ACTIVE Writing Group of the ACTIVE Investigators. Clopidogrel plus aspirin versus oral anticoagulation for atrial fibrillation in the Atrial fibrillation Clopidogrel Trial with Irbesartan for prevention of Vascular Events (ACTIVE W): a randomised controlled trial. Lancet 2006; 367(9526):1903-1912

6 Lip GY, Agnelli G. Edoxaban: a focused review of its clinical pharmacology. Eur Heart J 2014;35(28):1844-1855

7 Skjøth F, Larsen TB, Rasmussen LH, Lip GY. Efficacy and safety of edoxaban in comparison with dabigatran, rivaroxaban and apixaban for stroke prevention in atrial fibrillation. An indirect comparison analysis. Thromb Haemost 2014;111(5):981-988

8 Spinler SA, Shafir V. New oral anticoagulants for atrial fibrillation. Circulation 2012;126(1):133-137

9 Camm AJ, Lip GYH, De Caterina R, et al; ESC Committee for Practice Guidelines (CPG). 2012 focused update of the ESC Guidelines for the management of atrial fibrillation: an update of the 2010 ESC Guidelines for the management of atrial fibrillation. Developed with the special contribution of the European Heart Rhythm Association. Eur Heart J 2012;33(21):2719-2747
10 You JJ, Singer DE, Howard PA, et al; American College of Chest Physicians. Antithrombotic therapy for atrial fibrillation: Antithrombotic Therapy and Prevention of Thrombosis, 9th ed: American College of Chest Physicians Evidence-Based Clinical Practice Guidelines. Chest 2012;141(2, Suppl):e531S-e575S

11 Capodanno D, Angiolillo DJ. Management of antiplatelet and anticoagulant therapy in patients with atrial fibrillation in the setting of acute coronary syndromes or percutaneous coronary interventions. Circ Cardiovasc Interv 2014;7(1):113-124

12 Sørensen R, Gislason G. Triple antithrombotic therapy: risky but sometimes necessary. Rev Esp Cardiol (Engl Ed) 2014;67(3): 171-175

13 Mega J, Carreras ET. Antithrombotic therapy: triple therapy or triple threat? Hematology (Am Soc Hematol Educ Program) 2012; 2012:547-552

14 Yusuf S, Zhao F, Mehta SR, Chrolavicius S, Tognoni G, Fox KK; Clopidogrel in Unstable Angina to Prevent Recurrent Events Trial Investigators. Effects of clopidogrel in addition to aspirin in patients with acute coronary syndromes without ST-segment elevation. N Engl J Med 2001;345(7):494-502

15 Franchi F, Angiolillo DJ. Novel antiplatelet agents in acute coronary syndrome. Nat Rev Cardiol 2015;12(1):30-47

16 Giorgi MA, Cohen Arazi H, Gonzalez CD, Di Girolamo G. Beyond efficacy: pharmacokinetic differences between clopidogrel, prasugrel and ticagrelor. Expert Opin Pharmacother 2011;12(8): 1285-1295

17 Wallentin L, Becker RC, Budaj A, et al; PLATO Investigators. Ticagrelor versus clopidogrel in patients with acute coronary syndromes. N Engl J Med 2009;361(11):1045-1057

18 Wiviott SD, Braunwald E, McCabe $\mathrm{CH}$, et al; TRITON-TIMI 38 Investigators. Prasugrel versus clopidogrel in patients with acute coronary syndromes. N Engl J Med 2007;357(20):2001-2015

19 Lip GY, Windecker S, Huber K, et al; Document Reviewers. Management of antithrombotic therapy in atrial fibrillation patients presenting with acute coronary syndrome and/or undergoing percutaneous coronary or valve interventions: a joint consensus document of the European Society of Cardiology Working Group on Thrombosis, European Heart Rhythm Association (EHRA), European Association of Percutaneous Cardiovascular Interventions (EAPCI) and European Association of Acute Cardiac Care (ACCA) endorsed by the Heart Rhythm Society (HRS) and Asia-Pacific Heart Rhythm Society (APHRS). Eur Heart J 2014; 35(45):3155-3179

20 O'Gara PT, Kushner FG, Ascheim DD, et al; American College of Cardiology Foundation/American Heart Association Task Force on Practice Guidelines. 2013 ACCF/AHA guideline for the management of ST-elevation myocardial infarction: a report of the American College of Cardiology Foundation/American Heart Association Task Force on Practice Guidelines. Circulation 2013;127(4): e362-e425

21 Bonaca MP, Bhatt DL, Cohen M, et al; PEGASUS-TIMI 54 Steering Committee and Investigators. Long-term use of ticagrelor in patients with prior myocardial infarction. N Engl J Med 2015; 372(19):1791-1800

22 Mauri L, Kereiakes DJ, Yeh RW, et al; DAPT Study Investigators. Twelve or 30 months of dual antiplatelet therapy after drugeluting stents. N Engl J Med 2014;371(23):2155-2166

23 Palmerini T, Benedetto U, Bacchi-Reggiani L, et al. Mortality in patients treated with extended duration dual antiplatelet therapy after drug-eluting stent implantation: a pairwise and Bayesian network meta-analysis of randomised trials. Lancet 2015; 385(9985):2371-2382

24 Nieuwlaat R, Capucci A, Camm AJ, et al; European Heart Survey Investigators. Atrial fibrillation management: a prospective survey in ESC member countries: the Euro Heart Survey on Atrial Fibrillation. Eur Heart J 2005;26(22):2422-2434 
25 Nabauer M, Gerth A, Limbourg T, et al. The Registry of the German Competence NETwork on Atrial Fibrillation: patient characteristics and initial management. Europace 2009;11(4):423-434

26 Feit F, Voeltz MD, Attubato MJ, et al. Predictors and impact of major hemorrhage on mortality following percutaneous coronary intervention from the REPLACE-2 Trial. Am J Cardiol 2007;100(9): 1364-1369

27 Karjalainen PP, Porela P, Ylitalo A, et al. Safety and efficacy of combined antiplatelet-warfarin therapy after coronary stenting. Eur Heart J 2007;28(6):726-732

28 Ruiz-Nodar JM, Marín F, Hurtado JA, et al. Anticoagulant and antiplatelet therapy use in 426 patients with atrial fibrillation undergoing percutaneous coronary intervention and stent implantation implications for bleeding risk and prognosis. J Am Coll Cardiol 2008;51(8):818-825

29 Sørensen R, Hansen ML, Abildstrom SZ, et al. Risk of bleeding in patients with acute myocardial infarction treated with different combinations of aspirin, clopidogrel, and vitamin $\mathrm{K}$ antagonists in Denmark: a retrospective analysis of nationwide registry data. Lancet 2009;374(9706):1967-1974

30 Dewilde WJM, Oirbans T, Verheugt FWA, et al; WOEST study investigators. Use of clopidogrel with or without aspirin in patients taking oral anticoagulant therapy and undergoing percutaneous coronary intervention: an open-label, randomised, controlled trial. Lancet 2013;381(9872):1107-1115

31 Hansen ML, Sørensen R, Clausen MT, et al. Risk of bleeding with single, dual, or triple therapy with warfarin, aspirin, and clopidogrel in patients with atrial fibrillation. Arch Intern Med 2010; 170(16):1433-1441

32 Lamberts M, Olesen JB, Ruwald MH, et al. Bleeding after initiation of multiple antithrombotic drugs, including triple therapy, in atrial fibrillation patients following myocardial infarction and coronary intervention: a nationwide cohort study. Circulation 2012; 126(10):1185-1193

33 Goto K, Nakai K, Shizuta S, et al; CREDO-Kyoto Registry Cohort-2 Investigators. Anticoagulant and antiplatelet therapy in patients with atrial fibrillation undergoing percutaneous coronary intervention. Am J Cardiol 2014;114(1):70-78

34 Lamberts M, Gislason GH, Lip GYH, et al. Antiplatelet therapy for stable coronary artery disease in atrial fibrillation patients taking an oral anticoagulant: a nationwide cohort study. Circulation 2014;129(15):1577-1585

35 Lamberts M, Gislason GH, Olesen JB, et al. Oral anticoagulation and antiplatelets in atrial fibrillation patients after myocardial infarction and coronary intervention. J Am Coll Cardiol 2013;62(11): 981-989

36 Rha S-W. Duration of dual antiplatelet treatment in the era of next generation drug-eluting stents. World J Cardiol 2014;6(4): 148-153

37 Elmariah S, Mauri L, Doros G, et al. Extended duration dual antiplatelet therapy and mortality: a systematic review and meta-analysis. Lancet 2015;385(9970):792-798

38 Fiedler KA, Maeng M, Mehilli J, et al. Duration of Triple Therapy in Patients Requiring Oral Anticoagulation After Drug-Eluting Stent Implantation: The ISAR-TRIPLE Trial. J Am Coll Cardiol 2015; 65(16):1619-1629

39 Miller CS, Grandi SM, Shimony A, Filion KB, Eisenberg MJ. Meta-analysis of efficacy and safety of new oral anticoagulants (dabigatran, rivaroxaban, apixaban) versus warfarin in patients with atrial fibrillation. Am J Cardiol 2012;110(3):453-460

40 Lippi G, Favaloro EJ, Mattiuzzi C. Combined administration of antibiotics and direct oral anticoagulants: a renewed indication for laboratory monitoring? Semin Thromb Hemost 2014;40(7): 756-765

41 Alexander JH, Becker RC, Bhatt DL, et al; APPRAISE Steering Committee and Investigators. Apixaban, an oral, direct, selective factor Xa inhibitor, in combination with antiplatelet therapy after acute coronary syndrome: results of the Apixaban for Prevention of Acute Ischemic and Safety Events (APPRAISE) trial. Circulation 2009;119(22):2877-2885

42 Mega JL, Braunwald E, Wiviott SD, et al; ATLAS ACS 2-TIMI 51 Investigators. Rivaroxaban in patients with a recent acute coronary syndrome. N Engl J Med 2012;366(1):9-19

43 Oldgren J, Budaj $A$, Granger $C B$, et al; RE-DEEM Investigators. Dabigatran vs. placebo in patients with acute coronary syndromes on dual antiplatelet therapy: a randomized, double-blind, phase Il trial. Eur Heart J 2011;32(22):2781-2789

44 Oldgren J, Wallentin L, Alexander JH, et al. New oral anticoagulants in addition to single or dual antiplatelet therapy after an acute coronary syndrome: a systematic review and meta-analysis. Eur Heart J 2013;34(22):1670-1680

45 Connolly SJ, Ezekowitz MD, Yusuf S, et al; RE-LY Steering Committee and Investigators. Dabigatran versus warfarin in patients with atrial fibrillation. N Engl J Med 2009;361(12):1139-1151

46 Dans AL, Connolly SJ, Wallentin L, et al. Concomitant use of antiplatelet therapy with dabigatran or warfarin in the Randomized Evaluation of Long-Term Anticoagulation Therapy (RE-LY) trial. Circulation 2013;127(5):634-640

47 Alexander JH, Lopes RD, James S, et al; APPRAISE-2 Investigators. Apixaban with antiplatelet therapy after acute coronary syndrome. N Engl J Med 2011;365(8):699-708

48 Gibson CM, Mehran R, Bode C, et al. An open-label, randomized, controlled, multicenter study exploring two treatment strategies of rivaroxaban and a dose-adjusted oral vitamin $\mathrm{K}$ antagonist treatment strategy in subjects with atrial fibrillation who undergo percutaneous coronary intervention (PIONEER AF-PCI). Am Heart J 2015;169(4):472-8.e5

49 Sarafoff N, Martischnig A, Wealer J, et al. Triple therapy with aspirin, prasugrel, and vitamin $\mathrm{K}$ antagonists in patients with drug-eluting stent implantation and an indication for oral anticoagulation. J Am Coll Cardiol 2013;61(20):2060-2066

50 Savonitto S, Ferri M, Corrada E. Fatal bleedings with prasugrel as part of triple antithrombotic therapy. Rev Esp Cardiol (Engl Ed) 2014;67(3):225-226

51 January CT, Wann LS, Alpert JS, et al; American College of Cardiology/American Heart Association Task Force on Practice Guidelines. 2014 AHA/ACC/HRS guideline for the management of patients with atrial fibrillation: a report of the American College of Cardiology/American Heart Association Task Force on Practice Guidelines and the Heart Rhythm Society. J Am Coll Cardiol 2014; 64(21):e1-e76

52 Huber K, Airaksinen KJ, Cuisset T, Marín F, Rubboli A, Lip GYH. Antithrombotic therapy in patients with atrial fibrillation undergoing coronary stenting: similarities and dissimilarities between North America and Europe. Thromb Haemost 2011;106(4): 569-571 